\title{
Educational Programs to Build Resilience in Children, Adolescent or Youth with Disease or Disability: A Systematic Review
}

\author{
Martín García-Parra $^{1, *(D)}$, Francisca Negre ${ }^{2}(\mathbb{D})$ and Sebastià Verger ${ }^{2}$ (D) \\ 1 Department of Applied Pedagogy and Psychology of Education, University of the Balearic Islands, \\ 07122 Palma, Spain \\ 2 Hospital Pedagogy Lab, Department of Applied Pedagogy and Psychology of Education, Institute of \\ Research and Innovation in Education (IRIE), University of the Balearic Islands, 07122 Palma, Spain; \\ xisca.negre@uib.es (F.N.); s.verger@uib.es (S.V.) \\ * Correspondence: m.garcia@uib.es
}

Citation: García-Parra, M.; Negre, F.; Verger, S. Educational Programs to Build Resilience in Children, Adolescent or Youth with Disease or Disability: A Systematic Review. Educ. Sci. 2021, 11, 464. https:// doi.org/10.3390/educsci11090464

Academic Editor: Eila Jeronen

Received: 4 August 2021

Accepted: 22 August 2021

Published: 25 August 2021

Publisher's Note: MDPI stays neutral with regard to jurisdictional claims in published maps and institutional affiliations.

Copyright: (c) 2021 by the authors. Licensee MDPI, Basel, Switzerland. This article is an open access article distributed under the terms and conditions of the Creative Commons Attribution (CC BY) license (https:// creativecommons.org/licenses/by/ $4.0 /)$.

\begin{abstract}
Resilience is understood as interactive processes that strengthen the individual and the family in the face of the demands of adversity or vulnerable situation. Resilience is fostered from a psychopedagogical approach when practices are developed that assist in facing challenges positively, having life projects and developing academic potentialities. Thus, the objective of this systematic review of the literature is cartography programs that promote resilience in children, adolescents, or youths who are facing a challenging condition, such as a disease or disability. The PRISMA declaration was used to guide this systematic search. The databases consulted were Web of Science, Scopus, EBSCOhost, ERIC and Dialnet Plus. Open access articles were selected between 2016 and 2021. The selection resulted in 15 educational programs. The results indicate that there are several models for building resilience, such as ecosystem, family, community, and academic models. In conclusion, interdisciplinarity is a cross-cutting axis for enhancing resilience in vulnerable settings.
\end{abstract}

Keywords: resilience; educational programs; inclusive education; systematic review

\section{Introduction}

How to build a resilient character through interdisciplinary programs? The solution is not straightforward, but it is achievable. Thus, we must interpret resilience and its relationship from an interdisciplinary perspective. In respect of interpreting what is resilience, authors from a variety of disciplines have sought to clarify resilience by using several notions as a reference framework for their studies. This means that there is no single way to interpret resilience, even within the same field. According to Manciaux [1], these definitions often have only a weak consensus. The added nuances are substantial, and many of the terms used to define them in other disciplines of study are identical or quite similar. These different approximations and nuances within the same scientific discipline led to inconsistencies in concepts linked to resilience itself [2].

However, regardless of the discipline in which resilience is investigated, the definition utilized, or the approach taken by the researcher, the phenomenon resilience refers to the same idea. Thus, resilience is a reference framework for describing the positive aspects and mechanisms in an individual, group, material, or system that, when faced with a destabilizing and disruptive situation that threatens their integrity and stability, allows them to hold up, cope, recover, and emerge strengthened.

It is also agreed that the phenomenon can be looked at from an ecological point of view, meaning it can be used to refer to both individual characteristics and those of the context, as well as all the mechanisms used by the internal and external assets when facing adversity [3]. Furthermore, there are other factors that must be considered as influential in 
building resilience. These factors are related to family interactions and interactions with several professionals.

Regarding family interactions, it is vital to emphasize that the family is interpreted as a unit. To build resilience, family unit requires strong and committed strategies to meet the needs of a member with some vulnerability, but without abandoning the family integrity [4-6]. In addition, each family requires professional advice and guidance to achieve a comprehensive strengthening of resilience. Therefore, interdisciplinary field of resilience can be interpreted as the relationship of academic, health and psychosocial environment to reach a stable scenario that allows children, adolescents, youth, and their families develop internal strengths and capacities to face difficulties and set relationships whit others.

In perspective of this, interdisciplinary programs must emphasize the importance of teamwork from a holistic perspective. The holistic perspective postulate the diversity and complexity of a multiplicity of factors, before which, resilience is understood as dynamic. [7] Therefore, the holistic perspective is not aimed at developing simple formulas or recipes applicable to different contexts, but at offering strategies that, in face of complexity, can reveal synergies and dynamics to strengthen or build specific resilience processes [7]. This trend is the topic of this systematic review.

In addition, this interdisciplinary and holistic perspective of resilience is developing in educational settings. The school is a source of support for children, adolescents and young people exposed to several vulnerable or risk situations [1,7-9]. The focus of this paper is to explore how resilience is promoted for groups in vulnerable situations in different current educational programs. Before proceeding, it is essential to explain what is meant by resilience.

\section{The Concept of Resilience}

The concept of resilience refers to the capacity composed of the set of personal characteristics and the contexts of development of an individual and the set of internal and external mechanisms when facing adversity $[10,11]$. Thus, resilience is interpreted as the ability to respond positively, despite living in challenging or threatening circumstances.

This means holding out, facing, and reacting better than expected in a situation of risk, a traumatic event or adversity affecting the psycho-social integrity of an individual $[12,13]$. Others add that it is possible to come out of such situations strengthened, avoiding situations of risk, thus producing a better state $[14,15]$ From a purely individual level, resilience is considered as a characteristic of personality, the ability to adapt control depending on the circumstance [16].

Walsh $[17,18]$ according to whom resilience interactive processes that strengthen the individual and the family over time. With this family-centered approach, interdisciplinarity is vital to promote resilience, not only from an educational field, but also from a health and social perspective.

Masten [19] considered that resilience might depend on dynamic psychological processes such as the use of coping strategies (CS), and/or on personality factors configuring what she called resiliency. In relation to these two possibilities, on one side, some authors $[11,20,21]$ have shown the important role that the personality factors configuring resiliency play as potential determinants of resilience in adolescence. On the other side, resilience in adolescents depends in great degree on coping styles and strategies [6]. However, it is possible that the coping styles and the personality factors underlying resilience are related. If this were the case, it would be important to know the relative weight of each of these variables on resilience, as depending on the answer to this question, the implications for assessment and intervention would be different.

In sum, resilience is understood as a dynamic process, not as an absolute static or definitive quality [1]. It arises from a process of interaction which implies a positive personal and social adaptation of the individual despite exposure to risk [14]. It is evident in continuous interaction with the environment in which people develop and socialize [22]. 
It can vary according to the relationship with the context, requiring a reaction to a series of circumstances specific to each moment and everyone. It is applied when facing an adverse situation which could put personal and social stability at risk $[6,22,23]$. Greater resilience is shown on occasions when a person must put it into practice. However, the development of resilience is subject to the specific adjustment a person makes in an adverse situation [14].

This systematic review has the objective cartography and analyze programs which develop strategies to build resilience in children, adolescents, or youth with some vulnerability, such as disease or disability. Thus, this systematic review was carried out focused on educational, psychological and health programs, under an interdisciplinary perspective.

\section{Materials and Methods}

\subsection{Search Strategy}

Systematized review is a methodology that promotes transparency and rigor [24] and allows to identify trends and opportunities in future lines of research [25]. The Preferred Reporting Items for Systematic Reviews and Meta-analysis (PRISMA) guidelines [26] and the Framework ReSiste-CSH [27] were followed to conduct this systematic review. Moreover, this systematized review includes creating the database and the Visualizing Scientific Landscape (VOSviewer) analysis.

Between February and May of 2021, this systematic review was carried out. Databases used included: Scopus, Web of Science (WoS), ERIC, EBSCOhost (Psychology and Behavioral Sciences Collection, Academic Search Complete, APA PsycInfo, APA PsycArticles, E-Journals, eBook Collection, Social Work Abstracts, SocINDEX with Full Text) and Dialnet Plus. Descriptors and keywords were selected from DeCS (Health Sciences Descriptors) and ERIC thesaurus (Table 1).

Table 1. Keywords and descriptors.

\begin{tabular}{ccc}
\hline Keywords & DeCS & ERIC Thesaurus \\
\hline Resilience & Resilience, Psychological & $\begin{array}{c}\text { Resilience (Psychology) } \\
\text { Resilience (Academic) } \\
\text { Program/Intervention/Plan }\end{array}$ \\
\hline
\end{tabular}

Search strategies were adapted to the different academic databases, the final search equations presented in Table 2. In the search equations, used boolean operators (AND, OR) and truncations $\left(^{*}\right)$ for terms resilience, program, plan, and strategy. In addition, search was limited to the topic section (Title, Keyword and Abstracts). Inclusion criteria for the review were records from 2016 to 2021, records in Open Access from an account at the Library Service of the University of the Balearic Islands, article type, and English and Spanish language.

In total, 2451 articles were included for the evaluation phase [27]. All documents were uploaded to Mendeley's bibliographic manager. Furthermore, references were checked through the Endnote web manager.

Table 2. Search equations.

\begin{tabular}{|c|c|c|c|}
\hline Database & Search Equations & Date & Records \\
\hline Scopus & $\left(\left(\right.\right.$ resilienc $\left.^{*}\right)$ AND (program * OR plan * OR intervention OR strategy $\left.\left.{ }^{*}\right)\right)$ & 12 February 2021 & 421 \\
\hline WoS & $\left(\left(\right.\right.$ resilienc $\left.{ }^{*}\right)$ AND (program * OR plan * OR intervention OR strategy $\left.\left.{ }^{*}\right)\right)$ & 12 February 2021 & 810 \\
\hline EBSCOhost & $\left(\left(\right.\right.$ resilienc $\left.^{*}\right)$ AND (interventions or strategies or best practices) AND (interdisciplinary)) & 13 February 2021 & 475 \\
\hline ERIC & resilience AND (programs OR strategy OR plan OR intervention) & 13 February 2021 & 102 \\
\hline Dialnet Plus & $\left(\left(\right.\right.$ resilienc $\left.^{*}\right)$ AND (program * OR plan * OR intervention OR strategy *)) & 15 February 2021 & 643 \\
\hline
\end{tabular}

Note: There are truncations ${ }^{*}$ ) for terms: resilience, program, plan, and strategy. Using these truncations increased range of results in the bibliographic search. 


\subsection{Analysis Using VOSviewers}

Different visualization technologies have been applied for science mapping and bibliometric analysis in recent times. The main objectives of these visualizations are to highlight the dynamic and complex relationships between fields, authors, journals, organizations, countries, and key knowledge ideas.

This study made use of VOSviewer, a widely used application with an easy-to-use graphic interface for creating bibliometric networks of authors, publications, journals, organizations, and countries. Figure 1 depicts the study performed in VOSviewer using a Scopus database search. The Scopus results were used to analyze the cross-sectional nature of the keywords of the articles selected in the evaluation phase. It indicates how the descriptor psychological resilience is the most frequently used term in the results.

\section{象 Vosviewer}

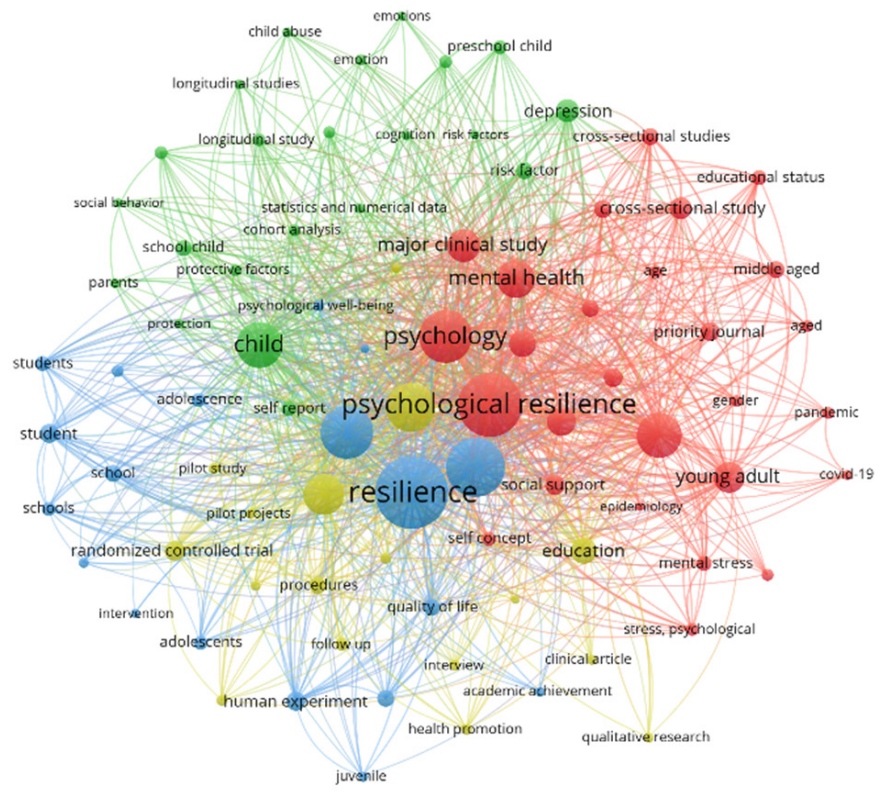

Figure 1. Bibliometric map of keywords of Scopus.

\subsection{Analytical Produce}

A peer review was conducted for the data extraction and confirmation procedure; all authors read the publications and selected the results to be included in the analysis phase. PRISMA Flowchart depicts the results of the articles' identification, screening, eligibility evaluation, and inclusion (Figure 2). This is still an essential component of report of completed systematic reviews [28]. Following the analysis phase, 15 papers were chosen for the synthesis phase. As a result, the analytical process was systematized through the creation of tables including the main bibliometric variables of the programs (Table 3).

In the database variable, 7 articles from WoS, 5 from Scopus, 1 from Eric, 1 form EBSCOhost and 1 from Dialnet Plus. Regarding publication by country, Spain has 5 records, followed by the USA (4), and the other countries with 1 record. Regarding language, 33\% are articles in Spanish and 67\% in English. Finally, Figure 3 shows how 2016 was the year with the highest number of articles and how in 2020 there was a rebound with 3 articles. 
Table 3. Bibliometric variables.

\begin{tabular}{|c|c|c|c|c|c|c|c|}
\hline Database & Year & Journal & Authors & Program & Method & Country & Language \\
\hline WoS & 2020 & Children & $\begin{array}{l}\text { Aggarwal, R. } \\
\text { Salamon, K.S. }\end{array}$ & $\begin{array}{l}\text { Outpatient Interdisciplinary } \\
\text { Pediatric Chronic Pain } \\
\text { Management Program [29] }\end{array}$ & QT & USA & EN \\
\hline EBSCOhost & 2019 & $\begin{array}{l}\text { Counselling Psychology } \\
\text { Quarterly }\end{array}$ & $\begin{array}{l}\text { Armstrong, L.L. } \\
\text { Desson, S. } \\
\text { St. John, E. } \\
\text { Watt, E. }\end{array}$ & D.R.E.A.M. Program [30] & MM & Canada & EN \\
\hline $\begin{array}{l}\text { Dialnet } \\
\text { Plus }\end{array}$ & 2016 & $\begin{array}{l}\text { Psicología y Educación: } \\
\text { Presente y Futuro }\end{array}$ & $\begin{array}{l}\text { Barrera, S.A. } \\
\text { Acle, G. } \\
\text { Martínez, L.M. }\end{array}$ & $\begin{array}{l}\text { Program for children with } \\
\text { language difficulties with the } \\
\text { risk/resilience ecosystem } \\
\text { model: [31] }\end{array}$ & QT & Mexico & SP \\
\hline Scopus & 2020 & $\begin{array}{c}\text { Electronic Journal of } \\
\text { Research in Educational } \\
\text { Psychology }\end{array}$ & $\begin{array}{l}\text { Cantero-García, M. } \\
\text { Garrido-Hernansaiz, H. } \\
\text { Alonso-Tapia, J. }\end{array}$ & $\begin{array}{l}\text { "Supérate. ¡No tires la toalla!" } \\
\text { Program [32] }\end{array}$ & QL & Spain & SP \\
\hline WoS & 2017 & Journal of Adolescence & $\begin{array}{c}\text { Dray, J. } \\
\text { Bowman, J. } \\
\text { Campbell, E. } \\
\text { Freund, M. } \\
\text { Hodder, R. } \\
\text { Wolfenden, L. } \\
\text { Richards, J. } \\
\text { Leane, C. } \\
\text { Green, S. } \\
\text { Lecathelinais, C. } \\
\text { Oldmeadow, C. } \\
\text { Attia, J. } \\
\text { Gillham, K. } \\
\text { Wiggers, J. }\end{array}$ & $\begin{array}{l}\text { Pragmatic school-based } \\
\text { universal intervention [33] }\end{array}$ & QT & Australia & EN \\
\hline Scopus & 2018 & Journal of Adolescence & $\begin{array}{l}\text { Jenkins, E.K. } \\
\text { Bungay, V. } \\
\text { Patterson, A. } \\
\text { Saewyc, E.M. } \\
\text { Johnson, J.L. }\end{array}$ & $\begin{array}{l}\text { Social Networking Action for } \\
\text { Resilience (SONAR) study [34] }\end{array}$ & MM & Canada & EN \\
\hline WoS & 2017 & $\begin{array}{l}\text { Social Science and } \\
\text { Medicine }\end{array}$ & $\begin{array}{l}\text { Li, X. } \\
\text { Harrison, S.E. } \\
\text { Fairchild, A.J. } \\
\text { Chi, P. } \\
\text { Zhao, J. } \\
\text { Zhao, G. }\end{array}$ & $\begin{array}{l}\text { Child-Caregiver-Advocacy } \\
\text { Resilience (ChildCARE) } \\
\text { intervention [35] }\end{array}$ & QT & $\begin{array}{l}\text { USA } \\
\text { China }\end{array}$ & EN \\
\hline Scopus & 2020 & $\begin{array}{l}\text { European Educational } \\
\text { Research Journal }\end{array}$ & $\begin{array}{c}\text { Morote, R. } \\
\text { Las Hayas, C. } \\
\text { Izco-Basurko, I. } \\
\text { Anyan, F. } \\
\text { Fullaondo, A. } \\
\text { Donisi, V. } \\
\text { Zwiefka, A. } \\
\text { Gudrun, D. } \\
\text { Ledertoug, M.M. } \\
\text { Olafsdottir, A.S. } \\
\text { Gabrielli, S. } \\
\text { Carbone, S. } \\
\text { Mazur, I. } \\
\text { Królicka-Deregowska, A. } \\
\text { Henrik Knoop, H. } \\
\text { Tange, N. } \\
\text { Kaldalóns, I. } \\
\text { Jónsdóttir, B. } \\
\text { González Pinto, A. } \\
\text { Hjemdal, O. }\end{array}$ & $\begin{array}{l}\text { UPRIGHT (Universal } \\
\text { Preventive Resilience } \\
\text { Intervention Globally } \\
\text { implemented in schools to } \\
\text { improve and promote mental } \\
\text { Health for Teenagers) [36] }\end{array}$ & MM & $\begin{array}{l}\text { Spain } \\
\text { Italy } \\
\text { Poland } \\
\text { Denmark } \\
\text { Iceland }\end{array}$ & EN \\
\hline WoS & 2016 & $\begin{array}{l}\text { Revista de Educación } \\
\text { Inclusiva Inclusive } \\
\text { Education Journal }\end{array}$ & Muñoz Garrido, V. & $\begin{array}{l}\text { Hospital classroom at the } \\
\text { CPEE Hospital Gregorio } \\
\text { Marañón, Madrid [37] }\end{array}$ & QL & Spain & SP \\
\hline ERIC & 2017 & $\begin{array}{l}\text { Revista Internacional de } \\
\text { Apoyo a la Inclusión, } \\
\text { Logopedia, Sociedad y } \\
\text { Multiculturalidad }\end{array}$ & Pérez Quinteros, L.E. & Proyecto Ángel [38] & MM & Chile & SP \\
\hline WoS & 2019 & Innovación Educativa & $\begin{array}{l}\text { Rascón, M.T. } \\
\text { Cabello, F. }\end{array}$ & $\begin{array}{l}\text { Educational innovation project: } \\
\text { Multimedia perspectives on } \\
\text { resilience and education [39] }\end{array}$ & MM & Spain & SP \\
\hline WoS & 2021 & PLoS ONE & $\begin{array}{l}\text { Rosen, J.G. } \\
\text { Phiri, L. } \\
\text { Chibuye, M. } \\
\text { Namukonda, E.S. } \\
\text { Mbizvo, M.T. } \\
\text { Kayeyi, N. }\end{array}$ & $\begin{array}{c}\text { Zambia Family (ZAMFAM) } \\
\text { Project [40] }\end{array}$ & QT & Zambia & EN \\
\hline
\end{tabular}


Table 3. Cont.

\begin{tabular}{|c|c|c|c|c|c|c|c|}
\hline Database & Year & Journal & Authors & Program & Method & Country & Language \\
\hline Scopus & 2016 & Family Process & Saltzman, W.R. & FOCUS Program [41] & QL & USA & EN \\
\hline WoS & 2016 & Anales de Psicología & $\begin{array}{c}\text { Sánchez-Hernández, Ó. } \\
\text { Méndez Carrillo, F.X. } \\
\text { Garber, J. }\end{array}$ & Penn Resiliency Program [42] & MM & Spain & EN \\
\hline Scopus & 2016 & Family Process & $\begin{array}{l}\text { Saul, J. } \\
\text { Simon, W. }\end{array}$ & $\begin{array}{l}\text { Summer Institute in Global } \\
\text { Mental Health and } \\
\text { Psychosocial Support [43] }\end{array}$ & QL & $\begin{array}{l}\text { USA } \\
\text { The } \\
\text { Netherlands }\end{array}$ & EN \\
\hline
\end{tabular}

Abbreviations: $\mathrm{QT}=$ Quantitative; $\mathrm{QL}=$ Qualitative; $\mathrm{MM}=$ Mixed Methods; EN = English; $\mathrm{SP}$ = Spanish; Len = Language.

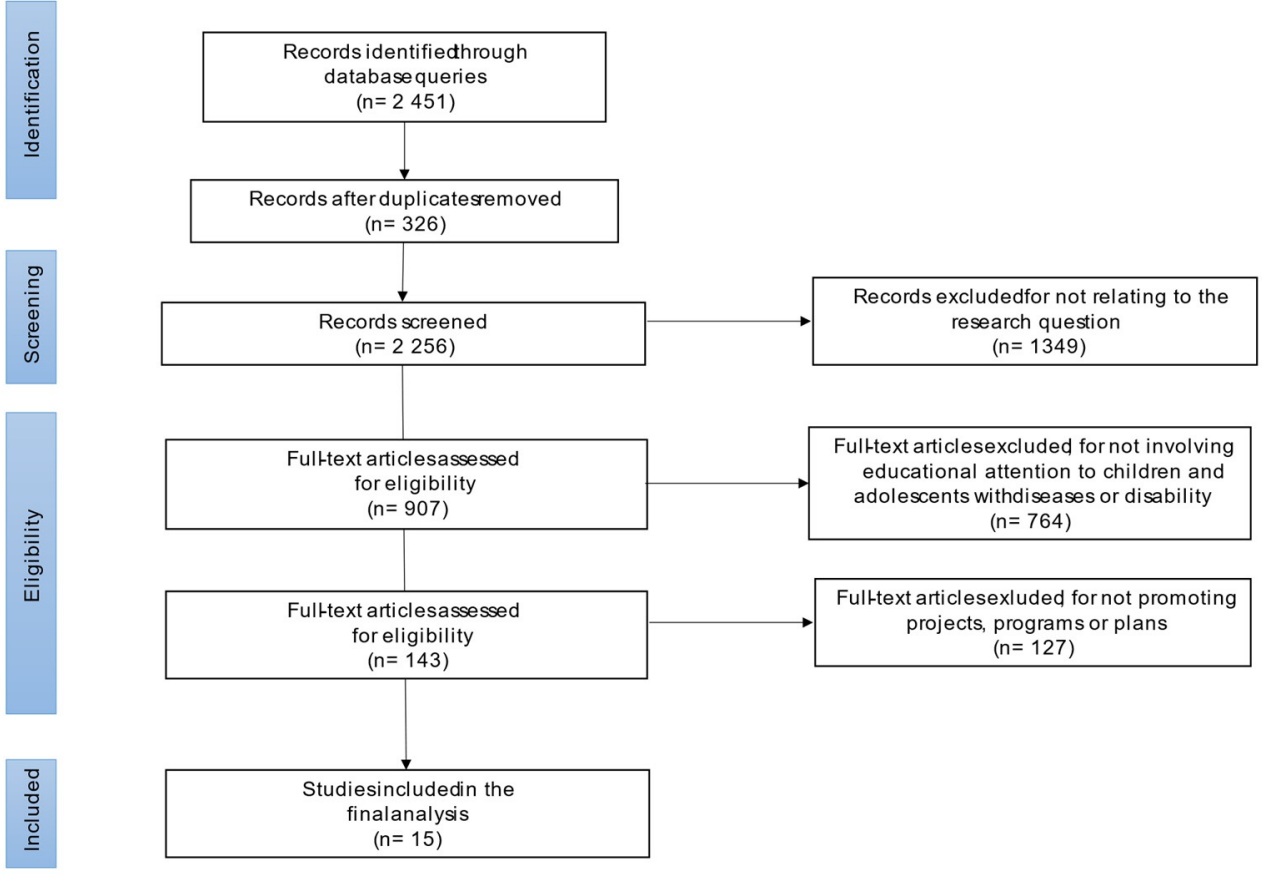

Figure 2. PRISMA flowchart of article selection produce.

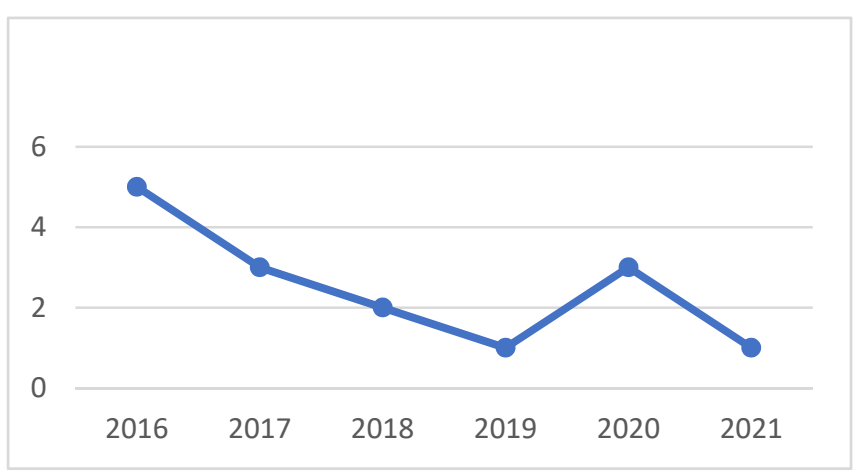

Figure 3. Evolution of articles per year.

\section{Results}

The results are presented below in relation to the goal of the investigation: cartography and analyze interdisciplinary programs which develop strategies to build resilience in children, adolescents, or youth with some vulnerability, such as disease or disability. In total, 15 programs matched the goal of this systematic review.

The programs were systematized by drawing up summarized tables with details of the main descriptive data (Table 4): Reference, Program, Objective, Participants, Design, Intervention, Measures, and Results. 
Table 4. Descriptive data of the studies.

\begin{tabular}{|c|c|c|c|c|c|c|c|}
\hline Reference & Program & Objective & Participants & Design & Intervention & Measures & Results \\
\hline $\begin{array}{l}\text { Aggarwal and } \\
\text { Salamon [29] }\end{array}$ & $\begin{array}{c}\text { Outpatient } \\
\text { Interdisciplinary } \\
\text { Pediatric } \\
\text { Chronic Pain } \\
\text { Management } \\
\text { Program }\end{array}$ & $\begin{array}{l}\text { Explore the risk } \\
\text { and resilience } \\
\text { factors that } \\
\text { contribute to } \\
\text { treatment } \\
\text { compliance and } \\
\text { functional } \\
\text { decline among } \\
\text { youth with } \\
\text { chronic pain } \\
\text { during } \\
\text { adolescence. }\end{array}$ & $\begin{array}{l}64 \text { adolescents } \\
\text { (11-18 years old) } \\
\text { diagnosed with } \\
\text { chronic pain } \\
\text { from a children's } \\
\text { hospital located } \\
\text { in the } \\
\text { northeastern } \\
\text { region of the } \\
\text { USA. }\end{array}$ & $\begin{array}{l}\text { Quasi- } \\
\text { experimental } \\
\text { study }\end{array}$ & $\begin{array}{c}\text { Psychological } \\
\text { interventions } \\
\text { based on } \\
\text { Cognitive- } \\
\text { Behavioral } \\
\text { Therapy (CBT) } \\
\text { with 6-10 } \\
\text { sessions for pain } \\
\text { and stress } \\
\text { management. }\end{array}$ & $\begin{array}{c}\text { Several } \\
\text { questionnaires. }\end{array}$ & $\begin{array}{l}\text { Findings indicate } \\
\text { that adolescent } \\
\text { resilience factors } \\
\text { (i.e., high pain } \\
\text { self-efficacy, high } \\
\text { pain acceptance) } \\
\text { may make } \\
\text { adolescents less } \\
\text { likely to comply } \\
\text { with treatment } \\
\text { overall. }\end{array}$ \\
\hline $\begin{array}{l}\text { Armstrong et al. } \\
\text { [30]. }\end{array}$ & $\begin{array}{l}\text { D.R.E.A.M. } \\
\text { Program }\end{array}$ & $\begin{array}{l}\text { The D.R.E.A.M. } \\
\text { (Developing } \\
\text { Resilience } \\
\text { through } \\
\text { Emotions, } \\
\text { Attitudes, and } \\
\text { Meaning) } \\
\text { program, } \\
\text { grounded in a } \\
\text { Second Wave } \\
\text { Positive } \\
\text { Psychology } \\
\text { approach called } \\
\text { R.E.A.L. } \\
\text { (Rational } \\
\text { Emotive } \\
\text { Attachment } \\
\text { Logotherapy). }\end{array}$ & $\begin{array}{l}45 \text { children, 6-12 } \\
\text { years old, who } \\
\text { are affiliated } \\
\text { with the } \\
\text { Association for } \\
\text { Bright Children } \\
\text { (ABC) Ottawa. }\end{array}$ & $\begin{array}{c}\text { Quasi- } \\
\text { experimental } \\
\text { study based on } \\
\text { Knowledge } \\
\text { Translation- } \\
\text { Integrated (KTI) } \\
\text { model. }\end{array}$ & $\begin{array}{c}\text { Educational } \\
\text { interventions } \\
\text { based on Social } \\
\text { and Emotional } \\
\text { Learning (SEL). }\end{array}$ & $\begin{array}{c}\text { Evaluation with } \\
\text { standards of } \\
\text { acceptability, } \\
\text { feasibility, } \\
\text { sustainability, } \\
\text { and credibility } \\
\text { [44] }\end{array}$ & $\begin{array}{l}\text { A positive } \\
\text { self-perception, a } \\
\text { sense of hope for } \\
\text { the future, and } \\
\text { an openness to } \\
\text { learning and } \\
\text { experiencing } \\
\text { new things were } \\
\text { the goals of the } \\
\text { program. }\end{array}$ \\
\hline Barrera et al. [31] & $\begin{array}{l}\text { Program for } \\
\text { children with } \\
\text { language } \\
\text { difficulties }\end{array}$ & $\begin{array}{c}\text { Program based } \\
\text { on } \\
\text { risk/resilience } \\
\text { ecosystem model } \\
\text { for children with } \\
\text { oral and written } \\
\text { language } \\
\text { difficulties. }\end{array}$ & $\begin{array}{l}6 \text { children with } \\
\text { oral and written } \\
\text { language } \\
\text { difficulties } \\
\text { between } 6 \text { and } 7 \\
\text { years old, his } \\
\text { parents and two } \\
\text { teachers. }\end{array}$ & $\begin{array}{l}\text { Quasi- } \\
\text { experimental } \\
\text { study }\end{array}$ & $\begin{array}{c}\text { Educational } \\
\text { interventions. }\end{array}$ & $\begin{array}{l}4 \text { stages: pretest, } \\
\text { intervention, } \\
\text { social validation } \\
\text { and } \\
\text { pretest-postest } \\
\text { assessment }\end{array}$ & $\begin{array}{l}\text { This work } \\
\text { emphasizes } \\
\text { effectiveness of } \\
\text { risk/resilience } \\
\text { ecosystem model } \\
\text { in the field of } \\
\text { Special } \\
\text { Education. }\end{array}$ \\
\hline $\begin{array}{l}\text { Cantero-García } \\
\text { et al. [32] }\end{array}$ & $\begin{array}{l}\text { "Supérate. ¡No } \\
\text { tires la toalla!" } \\
\text { Program }\end{array}$ & $\begin{array}{l}\text { Promote families' } \\
\text { ability to deal } \\
\text { with behavioral } \\
\text { issues, as well as } \\
\text { conflict methods } \\
\text { and parents' } \\
\text { emotional } \\
\text { regulation levels }\end{array}$ & $\begin{array}{l}61 \text { parents from } 7 \\
\text { Secondary } \\
\text { schools in } \\
\text { Madrid; } 41 \text { in } \\
\text { experimental } \\
\text { group and } 20 \text { in } \\
\text { control group. }\end{array}$ & $\begin{array}{l}\text { Non- } \\
\text { randomized } \\
\text { study }\end{array}$ & $\begin{array}{l}\text { Psychological } \\
\text { interventions }\end{array}$ & $\begin{array}{c}\text { Several } \\
\text { questionnaires to } \\
\text { evaluate various } \\
\text { items of this } \\
\text { program } \\
\text { (learning, } \\
\text { transfer, impact, } \\
\text { and perception } \\
\text { of quality). }\end{array}$ & $\begin{array}{l}\text { The program } \\
\text { produced a } \\
\text { significant } \\
\text { reduction in the } \\
\text { levels of anxiety, } \\
\text { depression as } \\
\text { well as an } \\
\text { improvement in } \\
\text { the perceived } \\
\text { family climate. }\end{array}$ \\
\hline Dray et al. [33] & $\begin{array}{l}\text { Pragmatic } \\
\text { school-based } \\
\text { universal } \\
\text { intervention }\end{array}$ & $\begin{array}{l}\text { Evaluate the } \\
\text { efficacy of a } \\
\text { universal, } \\
\text { school-based } \\
\text { intervention } \\
\text { focusing on } \\
\text { resilience } \\
\text { protective } \\
\text { variables in } \\
\text { reducing mental } \\
\text { health problems } \\
\text { in adolescents. }\end{array}$ & $\begin{array}{c}32 \text { secondary } \\
\text { schools within a } \\
\text { socio- } \\
\text { economically } \\
\text { disadvantaged } \\
\text { (students aged } \\
\text { 12-16 years) in } \\
\text { the Hunter New } \\
\text { England region } \\
\text { of New South } \\
\text { Wales (NSW), } \\
\text { Australia. }\end{array}$ & $\begin{array}{c}\text { Cluster } \\
\text { randomized } \\
\text { controlled trial }\end{array}$ & $\begin{array}{l}\text { Universal } \\
\text { school-based } \\
\text { intervention. }\end{array}$ & $\begin{array}{l}\text { Questionnaires } \\
\text { to measure } \\
\text { mental health } \\
\text { and internal and } \\
\text { external factors } \\
\text { of resilience in } \\
\text { students. } \\
\text { Additionally, } \\
\text { structured } \\
\text { interviews to } \\
\text { school staff. }\end{array}$ & $\begin{array}{l}\text { Study strengths } \\
\text { include a wide } \\
\text { range of } \\
\text { implementation } \\
\text { support } \\
\text { strategies, a } \\
\text { significant focus } \\
\text { on increasing } \\
\text { student } \\
\text { resilience, and a } \\
\text { large sample } \\
\text { size. }\end{array}$ \\
\hline Jenkins et al. [34] & $\begin{array}{c}\text { Social } \\
\text { Networking } \\
\text { Action for } \\
\text { Resilience } \\
\text { (SONAR) study }\end{array}$ & $\begin{array}{l}\text { Design a mental } \\
\text { health } \\
\text { promotion } \\
\text { intervention } \\
\text { based on } \\
\text { experience of } \\
\text { young people. }\end{array}$ & $\begin{array}{c}10 \text { youth } \\
\text { co-researchers } \\
\text { (YCRs) and } 344 \\
\text { students enrolled } \\
\text { in one Secondary } \\
\text { School (grades } 8 \\
\text { to 12) of a rural } \\
\text { community, } \\
\text { located in } \\
\text { North-Central } \\
\text { British Columbia, } \\
\text { Canada }\end{array}$ & $\begin{array}{c}\text { Experimental } \\
\text { Study based on } \\
\text { Community- } \\
\text { based } \\
\text { Knowledge } \\
\text { Translation } \\
\text { (CBKT) } \\
\text { framework. }\end{array}$ & $\begin{array}{l}\text { Mental health } \\
\text { promotion } \\
\text { interventions }\end{array}$ & $\begin{array}{l}\text { Mixed methods } \\
\text { approach (both } \\
\text { surveys and } \\
\text { qualitative } \\
\text { interviews) }\end{array}$ & $\begin{array}{l}\text { The SONAR } \\
\text { intervention } \\
\text { shows the } \\
\text { feasibility of } \\
\text { involving youth } \\
\text { in mental health } \\
\text { promotion as } \\
\text { well as a variety } \\
\text { of positive youth } \\
\text { development } \\
\text { benefits } \\
\text { connected with } \\
\text { this collaborative } \\
\text { strategy. }\end{array}$ \\
\hline
\end{tabular}


Table 4. Cont.

\begin{tabular}{|c|c|c|c|c|c|c|c|}
\hline Reference & Program & Objective & Participants & Design & Intervention & Measures & Results \\
\hline Li et al. [35] & $\begin{array}{c}\text { Child-Caregiver- } \\
\text { Advocacy } \\
\text { Resilience } \\
\text { (ChildCARE) } \\
\text { intervention }\end{array}$ & $\begin{array}{l}\text { Promote } \\
\text { resilience in } \\
\text { HIV / AIDS } \\
\text { people in rural } \\
\text { central China by } \\
\text { educating them } \\
\text { skills such as } \\
\text { positive thinking, } \\
\text { emotional } \\
\text { regulation, } \\
\text { coping, and } \\
\text { problem solving. }\end{array}$ & $\begin{array}{l}790 \text { Chinese } \\
\text { children (6-17 } \\
\text { years old) who } \\
\text { had a biological } \\
\text { father with } \\
\text { HIV / AIDS. } \\
\text { Children with } \\
\text { HIV infection } \\
\text { were excluded. }\end{array}$ & $\begin{array}{l}\text { Cluster } \\
\text { randomization- } \\
\text { controlled } \\
\text { trial }\end{array}$ & $\begin{array}{l}\text { Educational and } \\
\text { psychological } \\
\text { interventions }\end{array}$ & $\begin{array}{l}\text { Questionnaires } \\
\text { at baseline, } 6 \\
\text { months, and } 12 \\
\text { months that } \\
\text { included } \\
\text { demographic } \\
\text { and psychosocial } \\
\text { scales. }\end{array}$ & $\begin{array}{l}\text { The ChildCARE } \\
\text { intervention is } \\
\text { efficacious in } \\
\text { promoting } \\
\text { psychosocial } \\
\text { well-being of } \\
\text { children affected } \\
\text { by parental } \\
\text { HIV/AIDS in } \\
\text { rural China. }\end{array}$ \\
\hline Morote et al. [36] & $\begin{array}{c}\text { UPRIGHT } \\
\text { (Universal } \\
\text { Preventive } \\
\text { Resilience } \\
\text { Intervention } \\
\text { Globally } \\
\text { implemented in } \\
\text { schools to } \\
\text { promote mental } \\
\text { Health for } \\
\text { Teenagers) }\end{array}$ & $\begin{array}{l}\text { Promote mental } \\
\text { well-being by } \\
\text { enhancing } \\
\text { resilience } \\
\text { capacities in } \\
\text { young people }\end{array}$ & $\begin{array}{l}448 \text { Adolescents } \\
\text { between } 12 \text { and } \\
14 \text { years old, } 345 \\
\text { family members } \\
\text { and } 218 \text { school } \\
\text { staff and } \\
\text { teachers from } \\
\text { several schools } \\
\text { in Italy, } \\
\text { Denmark, Spain, } \\
\text { Poland, and } \\
\text { Iceland }\end{array}$ & $\begin{array}{l}\text { Mixed-methods } \\
\text { research process } \\
\text { combining a } \\
\text { survey study } \\
\text { with } \\
\text { participatory } \\
\text { group sessions } \\
\text { customized }\end{array}$ & $\begin{array}{c}\text { Educational } \\
\text { interventions. }\end{array}$ & $\begin{array}{c}\text { Participatory } \\
\text { sessions, survey } \\
\text { quantitative and } \\
\text { survey } \\
\text { qualitative }\end{array}$ & $\begin{array}{l}\text { The participants } \\
\text { agreed that in a } \\
\text { universal and } \\
\text { inclusive } \\
\text { program, each } \\
\text { member of the } \\
\text { school } \\
\text { community has a } \\
\text { concrete role in } \\
\text { fostering } \\
\text { resilience and } \\
\text { well-being for } \\
\text { all. }\end{array}$ \\
\hline $\begin{array}{c}\text { Muñoz Garrido } \\
\text { [37] }\end{array}$ & $\begin{array}{c}\text { Hospital } \\
\text { classroom at the } \\
\text { CPEE Hospital } \\
\text { Gregorio } \\
\text { Marañón, } \\
\text { Madrid }\end{array}$ & $\begin{array}{l}\text { Analysis of the } \\
\text { program based } \\
\text { on resilience in } \\
\text { the hospital } \\
\text { classroom }\end{array}$ & $\begin{array}{l}\text { School unit at } \\
\text { the Gregorio } \\
\text { Marañón } \\
\text { Hospital, to care } \\
\text { for children } \\
\text { admitted for } \\
\text { poliomyelitis. }\end{array}$ & $\begin{array}{l}\text { Descriptive and } \\
\text { interpretative- } \\
\text { symbolic } \\
\text { study }\end{array}$ & $\begin{array}{c}\text { Educational } \\
\text { interventions. }\end{array}$ & $\begin{array}{c}\text { Satisfaction } \\
\text { survey }\end{array}$ & $\begin{array}{l}\text { Students will be } \\
\text { more motivated } \\
\text { if their teachers } \\
\text { provide them } \\
\text { with an } \\
\text { appropriate } \\
\text { learning } \\
\text { environment, } \\
\text { including } \\
\text { techniques, } \\
\text { methodology, } \\
\text { and humanism. }\end{array}$ \\
\hline $\begin{array}{c}\text { Pérez Quinteros } \\
{[38]}\end{array}$ & Proyecto Ángel & $\begin{array}{l}\text { Analyze whether } \\
\text { there are any } \\
\text { strategies that } \\
\text { favor the } \\
\text { development of } \\
\text { resilience in } \\
\text { students with } \\
\text { attention deficit } \\
\text { hyperactivity } \\
\text { disorder } \\
\text { (ADHD). }\end{array}$ & $\begin{array}{l}\text { Students with } \\
\text { ADHD of the } \\
\text { second level of } \\
\text { Basic General } \\
\text { Education who } \\
\text { participated in a } \\
\text { local service } \\
\text { project called } \\
\text { "Angel Project" } \\
\text { in Chile. }\end{array}$ & $\begin{array}{l}\text { Action-research } \\
\text { study framed in } \\
\text { an interpretative } \\
\text { paradigm }\end{array}$ & $\begin{array}{l}\text { Educational and } \\
\text { psychological } \\
\text { interventions }\end{array}$ & $\begin{array}{l}\text { Mixed methods } \\
\text { (participant } \\
\text { observation, } \\
\text { interviews, a } \\
\text { group } \\
\text { discussion, and a } \\
\text { Likert scale) }\end{array}$ & $\begin{array}{l}\text { Strategies that } \\
\text { build resilience } \\
\text { refer to } \\
\text { enhancement of } \\
\text { self-esteem in } \\
\text { collaborative } \\
\text { work, and } \\
\text { development of } \\
\text { communication } \\
\text { skills and } \\
\text { emotional } \\
\text { expression. }\end{array}$ \\
\hline $\begin{array}{l}\text { Rascón and } \\
\text { Cabello [39] }\end{array}$ & $\begin{array}{l}\text { Multimedia } \\
\text { perspectives on } \\
\text { resilience and } \\
\text { education project }\end{array}$ & $\begin{array}{l}\text { Improve the } \\
\text { resilience of } \\
\text { children and } \\
\text { youth in } \\
\text { vulnerable } \\
\text { situations }\end{array}$ & $\begin{array}{c}268 \text { university } \\
\text { students and } \\
\text { Children and } \\
\text { youth in } \\
\text { vulnerable } \\
\text { situations in } \\
\text { Malaga (Spain). }\end{array}$ & $\begin{array}{l}\text { Project-based } \\
\text { cooperative } \\
\text { learning and } \\
\text { Service-learning }\end{array}$ & $\begin{array}{c}\text { Socio- } \\
\text { educational } \\
\text { interventions }\end{array}$ & $\begin{array}{l}\text { Qualitative } \\
\text { evaluations }\end{array}$ & $\begin{array}{l}77 \text { audiovisual } \\
\text { pieces were } \\
\text { created for } 27 \\
\text { partnering } \\
\text { entities in the } \\
\text { process of } \\
\text { resilience and } \\
\text { inclusion of } \\
\text { vulnerable } \\
\text { groups. }\end{array}$ \\
\hline Rosen et al. [40] & $\begin{array}{l}\text { Zambia Family } \\
\text { (ZAMFAM) } \\
\text { Project }\end{array}$ & $\begin{array}{l}\text { Strengthening } \\
\text { the household's } \\
\text { capacity to meet } \\
\text { the needs of } \\
\text { people living } \\
\text { with or affected } \\
\text { by HIV, as well } \\
\text { as improving the } \\
\text { well-being of the } \\
\text { child and } \\
\text { caregivers. }\end{array}$ & $\begin{array}{l}544 \text { Adolescents } \\
\text { living with HIV } \\
\text { (ALHIV) aged } \\
\text { 5-17 years and } \\
\text { their adult } \\
\text { caregivers in } \\
\text { Zambia }\end{array}$ & $\begin{array}{l}\text { Prospective } \\
\text { cohort study }\end{array}$ & $\begin{array}{c}\text { Multilevel } \\
\text { interventions } \\
\text { (education, } \\
\text { psychosocial, } \\
\text { economic, and } \\
\text { clinical services) }\end{array}$ & $\begin{array}{l}\text { Structured } \\
\text { interviews and } \\
\text { Poisson } \\
\text { regression with } \\
\text { generalized } \\
\text { estimating } \\
\text { equations } \\
\text { measured } \\
\text { one-year } \\
\text { changes }\end{array}$ & $\begin{array}{c}\text { Significant } \\
\text { improvements in } \\
\text { caregivers' } \\
\text { financial capacity } \\
\text { were observed } \\
\text { among house- } \\
\text { holds receiving } \\
\text { ZAMFAM } \\
\text { services, with } \\
\text { few changes in } \\
\text { health or } \\
\text { wellbeing among } \\
\text { ALHIV. }\end{array}$ \\
\hline
\end{tabular}


Table 4. Cont.

\begin{tabular}{|c|c|c|c|c|c|c|c|}
\hline Reference & Program & Objective & Participants & Design & Intervention & Measures & Results \\
\hline Saltzman [41] & FOCUS Program & $\begin{array}{l}\text { Promote family } \\
\text { resilience } \\
\text { through } \\
\text { communication } \\
\text { and make sense } \\
\text { of traumatic } \\
\text { experiences. }\end{array}$ & $\begin{array}{l}\text { Families exposed } \\
\text { to significant } \\
\text { levels of stress or } \\
\text { loss who may be } \\
\text { at risk for } \\
\text { psychological } \\
\text { disturbance }\end{array}$ & $\begin{array}{l}\text { Longitudinal } \\
\text { study }\end{array}$ & $\begin{array}{l}\text { Psychoeducational } \\
\text { interventions } \\
\text { focused on } \\
\text { families }\end{array}$ & Mixed methods & $\begin{array}{c}\text { A structured } \\
\text { family approach, } \\
\text { creating shared } \\
\text { goals, } \\
\text { strengthening } \\
\text { communication, } \\
\text { and practicing } \\
\text { specific skills } \\
\text { that promote } \\
\text { family resilience. }\end{array}$ \\
\hline $\begin{array}{c}\text { Sánchez- } \\
\text { Hernández, et al. } \\
\text { [42] }\end{array}$ & $\begin{array}{l}\text { Penn Resiliency } \\
\text { Program }\end{array}$ & $\begin{array}{l}\text { Study the } \\
\text { effectiveness of a } \\
\text { cognitive- } \\
\text { behavioral } \\
\text { intervention } \\
\text { inspired by the } \\
\text { Penn Resiliency } \\
\text { Program for the } \\
\text { prevention of } \\
\text { depression in } \\
\text { students from } \\
\text { primary } \\
\text { education. }\end{array}$ & $\begin{array}{c}25 \text { students, } \\
10-12 \text { years old, } \\
\text { selected from } 185 \\
\text { schoolchildren in } \\
\text { grades } 5 \text { and } 6 \text { of } \\
\text { Primary } \\
\text { education. }\end{array}$ & $\begin{array}{l}\text { Randomized } \\
\text { experimental } \\
\text { study. } \\
\text { Participants } \\
\text { were randomly } \\
\text { assigned to the } \\
\text { experimental } \\
\text { group } \\
\text { (preventive } \\
\text { intervention) } \\
\text { and control } \\
\text { (waiting list }\end{array}$ & $\begin{array}{l}\text { Cognitive- } \\
\text { behavioral } \\
\text { interventions. }\end{array}$ & $\begin{array}{c}\text { A mixed } 2 \times 2 \\
\text { factorial design } \\
\text { with an inter } \\
\text { factor } \\
\text { (prevention } \\
\text { program; } \\
\text { waiting list) and } \\
\text { an intra factor } \\
\text { (pretest, } \\
\text { posttest). }\end{array}$ & $\begin{array}{l}\text { Results indicated } \\
\text { that there was } \\
\text { significant } \\
\text { improvement } \\
\text { from pre-test to } \\
\text { post-test in the } \\
\text { experimental } \\
\text { group for } \\
\text { children with } \\
\text { "high depressive } \\
\text { symptoms" } \\
\text { compared with } \\
\text { controls. }\end{array}$ \\
\hline $\begin{array}{c}\text { Saul and Simon } \\
\text { [43] }\end{array}$ & $\begin{array}{l}\text { Summer } \\
\text { Institute in } \\
\text { Global Mental } \\
\text { Health and } \\
\text { Psychosocial } \\
\text { Support }\end{array}$ & $\begin{array}{l}\text { Strengthening } \\
\text { the capacity of } \\
\text { families, } \\
\text { communities, } \\
\text { and } \\
\text { organizations to } \\
\text { address mental } \\
\text { health issues and } \\
\text { promote } \\
\text { psychosocial } \\
\text { well-being. }\end{array}$ & $\begin{array}{l}24 \text { professionals } \\
\text { (several mental } \\
\text { health } \\
\text { professionals, a } \\
\text { psychiatrist, a } \\
\text { psychologist, a } \\
\text { human rights } \\
\text { practitioner, and } \\
\text { an ethnomusicol- } \\
\text { ogist) }\end{array}$ & $\begin{array}{l}\text { Randomized } \\
\text { experimental } \\
\text { study }\end{array}$ & $\begin{array}{l}\text { Training } \\
\text { program with } \\
\text { educational, } \\
\text { psychosocial, } \\
\text { and health } \\
\text { interventions }\end{array}$ & $\begin{array}{l}\text { Qualitative } \\
\text { interpretation of } \\
\text { experience in the } \\
\text { Summer } \\
\text { Institute }\end{array}$ & $\begin{array}{l}\text { The Summer } \\
\text { Institute } \\
\text { provided } \\
\text { training to } \\
\text { promote a } \\
\text { systems-oriented } \\
\text { resilience } \\
\text { approach in the } \\
\text { field of Global } \\
\text { Mental Health } \\
\text { and Psychosocial } \\
\text { Support } \\
\text { (GMHPSS) }\end{array}$ \\
\hline
\end{tabular}

\section{Discussion}

This review offers a comprehensive summary of the 15 educational programs to promote resilience in the field of care for children, adolescents, or young people, including their families, with a situation of vulnerability due to a disease or disability. By summarizing the information in the articles, an up-to-date overview of current knowledge in this field is obtained, focusing on the results provided by different resilience-building interventions with educational and interdisciplinary programs. However, these results must be treated with caution.

Furthermore, this systematized review makes it possible to map various educational programs focused on resilience with different intervention and design models. On the one hand, the family-centered model is present in $46 \%$ of the programs [29,31,32,36,37,40,41]. This approach considers that families are a fundamental pillar in making informed decisions, thus being the center from which the promotion of the quality of life of children and adolescents begins. Thus, family resilience is understood as the interactive processes that strengthen the individual and the family in the face of the demands of the disease [17,45]. For example, "Supérate. ¡No tires la toalla!" Program used group techniques such as the case study method, guided discussion, role play, activities, and commitments according to the experimental methodology to assist parents learn or improve their coping skills for stress and self-regulation of their emotions, thereby increasing their resilience. This program consisted of 10 group sessions of 90 min per week divided into two parts: first, family conflict resolution strategies (sessions 1-6); second, coping strategies and improving levels of resilience (sessions 7-10).

Another program, FOCUS [41] provided family psychoeducation and developmental guidance through shared family narratives using the timeline technique. In this program, interventions were carried out in eight sessions: the first two with the parents, the second two with the children, a fifth session with the parents to prepare for the family sessions, 
and then a series of three family sessions. Hence, having a family approach allows integral care, which not only meets the needs of the child, adolescent or youth, but also the needs of the family that, in addition to contributing to the physical and emotional health of its members, the family can be potential victim of the effects of the disease [46].

With this family-based approach to integral care, interdisciplinarity is vital to promote resilience. Therefore, interdisciplinarity of several fields, such as educational, psychological, social and health, will allow an ecosystem approach to resilience. This review makes it possible to detect various programs that stand out for an ecosystem and interdisciplinary approach. Aggarwal and Salamon [29] described an intervention in the hospital setting from an interdisciplinary approach. In this program, each adolescent who entered the program was evaluated by a medical provider, a psychologist, and a physical or occupational therapist. Her families also were given treatment recommendations, including individual, family, and group psychotherapy.

In Li et al. [35], a community vision of resilience is promoted in children of parents affected by HIV in a rural context. The intervention consisted of three levels: child, caregiver, and community. The child intervention included $20 \mathrm{~h}$ of facilitator-guided programming given in 10 peer-group sessions to increase resilience by developing skills (positive thinking, emotional regulation, coping, and problem solving). At the caregiver level, five sessions were organized with the goal of improving positive parenting skills and participating in personal care and seeking support. At the community level, a series of community activities were organized to promote cohesion. Rosen et al. [40] conducted a similar project (ZAMFAM) in which they promoted community resilience of adolescents with HIV and their families in the context of Zambia ZAMFAM's multilevel interventions supplement an integral package of health and social support services across five domains: HIV care and treatment, Parenting, Food security, Household economic strengthening, and Psychosocial support. In Muñoz Garrido [37], the need and importance of interdisciplinary work in hospital classrooms is evident. In this program of Hospital Pedagogy, educational interventions were supported by a coordinated multiprofessional intervention adapting interventions at level of curricular competence of the students, their diagnosis, the entrance phase, and their response capacity. Thus, integral and ecosystem care for children, adolescents and youth with diseases and or disabilities is based on interdisciplinarity [47].

Regarding the educational context, resilience is understood as the achievement of good educational results despite the adversity of the students [48]. Thus, a school based on resilience is one that develops educational practices that help students to face difficulties in a positive way, to have life projects and to develop their potentialities. This review shows that various programs focus on the academic model of resilience. The following programs with educational interventions are described.

DREAM Program [30] makes it possible to improve the academic agency of students following the psychological model called R.E.A.L. (Rational Emotive Attachment Logotherapy). DREAM is a SEL program that consisted of 10 brief units with administration times ranging from 5 to $30 \mathrm{~min}$. Each of the units included an original song and a hands-on activity as a teaching tool. In Barrera et al. [31] resilience was promoted in children between 6 and 7 years old with some oral and writing language difficulties. This program worked with the group of six children during 40 sessions twice a week. In addition, three sessions were dedicated to parents to promote the correct practice of the elements of the language. Additionally, three meetings were held with the teachers to share information about the performance and development of the participating children.

Dray et al. [33] postulated a strategy based on universal school to promote resilience and mental health for adolescents. This program designed 16 strategies to address internal factors (cooperation, communication, empathy, self-efficacy) or external resilience protective factor (school support, school meaningful participation, peer caring relationships). Schools were required to implement $9 \mathrm{~h}$ of resilience-focused content in a minimum of three of the following key learning areas (KLAs): English; Math; Science; History and Geography, 
or Personal Development, Health and Physical Education (PDHPE). Furthermore, schools received support from an interdisciplinary school intervention team.

UPRIGHT program [36] was implemented to improve and promote adolescent mental health. In this program, teachers were asked to implement 18 sessions (each session at least $40 \mathrm{~min}$ ) with adolescents. Sessions included (a) 1 session to present the program; (b) 14 sessions dedicated to each skill of the Coping, Efficacy and Socio-Emotional Learning components; and (c) 3 sessions dedicated to the skills, concerns, or preferred activities suggested because of the co-creation process in their countries.

Angel project [38] was aimed at students with ADHD to build their resilient personality. This project created intervention strategies in five categories: emotional identification, identify, self-esteem, communication skills, and teamwork. This program consisted of 15 sessions: five were assigned to the category of feelings and emotions, three to the category of identity, three to the category of self-esteem, three to the category of communication skills and two to the category of teamwork.

Multimedia perspectives project [39] proposed a socio-educational interventions through an edu-communicative approach in favor of the resilience of children and youth in vulnerable situation. This initiative brought together instructors and students of education, communications, and psychology, together with social entities and a social educator to cooperatively create a series of multimedia videos and a documentary short film, to disseminate and reflect on the processes of social exclusion and childhood resilience.

Penn Resiliency Program [42] aimed to prevent depression in elementary school students. In this program, twelve sessions of two hours each were used to deliver the cognitive-behavioral intervention in a group setting. According to the handbook, the group leader conducted two sessions per week, and students were given an activity book to use during those sessions. Session models consisted of four elements: (a) AdversityBeliefs-Consequences (ABC model); (b) Changes to the explanatory style; (c) Questioning and de-catastrophizing; (d) Resolution of interpersonal problems. For organizational team development and stress management, the program included a blend of didactic presentations, hands-on interactive exercises, and case studies.

The Summer Institute [43] fostered an ecosystem immersion of community resilience in the field of public mental health. This program was a two models' immersion: a multisystemic, strength-based viewpoint was used in Module I to introduce participants to clinical and community approaches to mental health and psychosocial well-being promotion. Module 2 focused on psychosocial and clinical approaches targeting populations at risk for common mental health conditions.

Finally, SONAR study [34] promoted community resilience through youth co-researchers (YCRs). In the rural community of SONAR study, youth were not exposed to opportunities or engaged in discussion about possibilities for their future. Interventions consisted of several group meeting led by the YCRs. During meetings, the youth identified that they wanted to create a web-app (called SONAR) that would be simple and easy to use. The web-app was viewed as a platform for sparking dialogue about the needs of youth in this community and initiating further opportunities for positive engagement.

The limitations of this systematized review were, on the one hand, the search for equations of generic terms to find studies or programs related to our research objective; on the other hand, the term resilience is used in different areas and fields, this resulted in the selection of the articles being more careful, trying to ensure that the programs were appropriate to the research question.

\section{Conclusions}

This systematic review provides evidence to support the design and implementation of educational programs to enhance or strengthen resilience in vulnerable groups. Vulnerable groups need integral care; therefore, first, it is suggested that educational programs should strengthen interdisciplinary interventions. Furthermore, secondly, it is suggested that these educational interventions integrate the family and social context from an ecological and 
holistic model of resilience. Thus, this review can act as a guide to generate more inclusive and resilience-building educational programs. Hence, programs to promote resilience in children, adolescents, or young people with a situation of vulnerability due to a disease or disability must have a transversal axis based on interdisciplinarity.

On the other hand, it is essential to emphasize future research on two critical aspects: (a) how to build or create interprofessional commitments that generate good teaching practices to promote resilience, and (b) how to evaluate and transfer these interprofessional commitments to offer cohesion to a resilient character in children, adolescents, youths, and their families with some situation of disease or disability.

Author Contributions: Conceptualization, M.G.-P., S.V. and F.N.; methodology, M.G.-P., S.V. and F.N.; formal analysis, M.G.-P., S.V. and F.N.; resources, M.G.-P., S.V. and F.N.; data curation, M.G.-P.; writing-original draft preparation, M.G.-P.; writing—review and editing, S.V. and F.N.; supervision, M.G.-P., S.V. and F.N.; project administration, M.G.-P., S.V. and F.N.; funding acquisition, S.V. and F.N. All authors have read and agreed to the published version of the manuscript.

Funding: This research was funded by the Agencia Estatal de Investigación (AEI) and the Ministry of Science and Innovation (Spain), which finances a pre-doctoral FPI contract, grant number BES2017-083041.

Conflicts of Interest: The authors declare no conflict of interest.

\section{References}

1. Manciaux, M. La Resiliencia: Resistir y Rehacerse; Editorial Gedisa: Barcelona, Spain, 2010.

2. Windle, G. What is resilience? A review and concept analysis. Rev. Clin. Gerontol. 2011, 21, 152-169. [CrossRef]

3. Ungar, M. The social ecology of resilience: Addressing contextual and cultural ambiguity of a nascent construct. Am. J. Orthopsychiatry 2011, 81, 1-17. [CrossRef]

4. Rolland, J.S.; Walsh, F. Facilitating family resilience with childhood illness and disability. Curr. Opin. Pediatr. 2006, 18, 527-538. [CrossRef]

5. Walsh, F. Strengthening Family Resilience, 3rd ed.; Guilford Press: New York, NY, USA, 2016.

6. Henderson Grotberg, E. La Resiliencia en el Mundo de Hoy. Cómo Superar Las Adversidades; Editorial Gedisa: Barcelona, Spain, 2013.

7. Forés Miravalles, A.; Grané Ortega, J. La Resiliencia en Entornos Socioeducativos; Narcea S.A. De Ediciones: Madrid, Spain, 2018.

8. Barankin, T.; Khanlou, N. Growing Up Resilient: Ways to Build Resilience in Children and Youth; Centre for Addition and Mental Health: Toronto, ON, Canada, 2007.

9. Puig, G.; Rubio, J.L. Manual de Resiliencia Aplicada; Editorial Gedisa: Barcelona, Spain, 2011.

10. Masten, A.S. Resilience in developing systems: The promise of integrated approaches. Eur. J. Dev. Psychol. 2016, 13, 297-312. [CrossRef]

11. Ungar, M. The Social Ecology of Resilience: A Handbook of Theory and Rractice; Springer Science + Business Media: New York, NY, USA, 2012.

12. Garmezy, N. Resiliency and Vulnerability to Adverse Developmental Outcomes Associated With Poverty. Am. Behav. Sci. 1991, 34, 416-430. [CrossRef]

13. Masten, A.S. Ordinary magic: Resilience processes in development. Am. Psychol. 2001, 56, 227-238. [CrossRef]

14. Luthar, S.S.; Cicchetti, D.; Becker, B. The Construct of Resilience: A Critical Evaluation and Guidelines for Future Work. NIH Public Access 2000, 71, 543-562. [CrossRef]

15. Werner, E.E. Risk, resilience, and recovery: Perspectives from the Kauai Longitudinal Study. Dev. Psychopathol. 1993, 5, 503-515. [CrossRef]

16. Brooks, R.; Goldstein, S. The Power of Resilience; McGraw-Hill Companies: New York, NY, USA, 2003.

17. Rolland, J.S.; Walsh, F. Systemic Training for Healthcare Professionals: The Chicago Center for Family Health Approach. Fam. Process 2005, 44, 283-301. [CrossRef] [PubMed]

18. Walsh, F. Family resilience: A developmental systems framework. Eur. J. Dev. Psychol. 2016, 13, 313-324. [CrossRef]

19. Masten, A.S. Resilience in developing systems: Progress and promise as the fourth wave rises. Dev. Psychopathol. 2007, 19, 921-930. [CrossRef]

20. Ungar, M. Systemic resilience: Principles and processes for a science of change in contexts of adversity. Ecol. Soc. $2018,23$. [CrossRef]

21. Barudy, J.; Dantagnan, M. Los Buenos Tratos a la Infancia; Editorial Gedisa: Barcelona, Spain, 2005.

22. Vanistendael, S.; Lecomte, J. La Felicidad es Posible. Despertar en Niños Maltratados la Confianza en sí Mismos: Construir la Resiliencia; Editorial Gedisa: Barcelona, Spain, 2002.

23. Cyrulnik, B.; Capdevila, C. Diálogos. Cyrulnik y Capdevila; Editorial Gedisa: Barcelona, Spain, 2017.

24. Hart, C. Doing a Literature Review: Releasing the Social Science Research Imagination; SAGE: London, UK, 2008. 
25. Manchado Garabito, R.; Tamames Gómez, S.; López González, M.; Mohedano Macías, L.; D’Agostino, M.; Veiga de Cabo, J. Revisiones Sistemáticas Exploratorias. Med. Segur. Trab. 2009, 55, 12-19. [CrossRef]

26. Liberati, A.; Altman, D.G.; Tetzlaff, J.; Mulrow, C.; Gøtzsche, P.C.; Ioannidis, J.P.A.; Clarke, M.; Devereaux, P.; Kleijnen, J.; Moher, D. The PRISMA statement for reporting systematic reviews and meta-analyses of studies that evaluate health care interventions: Explanation and elaboration. PLoS Med. 2009, 6, e1000100. [CrossRef] [PubMed]

27. Codina, L. Revisiones Bibliográficas Sistematizadas. Procedimientos Generales y Framework para Ciencias Humanas y Sociales; Universitat Pompeu Fabra: Barcelona, Spain, 2018.

28. Urrútia, G.; Bonfill, X. PRISMA declaration: A proposal to improve the publication of systematic reviews and meta-analyses. Med. Clin. 2010, 135, 507-511. [CrossRef]

29. Aggarwal Dutta, R.; Salamon, K.S. Risk and Resilience Factors Impacting Treatment Compliance and Functional Impairment among Adolescents Participating in an Outpatient Interdisciplinary Pediatric Chronic Pain Management Program. Children 2020, 7, 247. [CrossRef]

30. Armstrong, L.L.; Desson, S.; St John, E.; Watt, E. The D.R.E.A.M. program: Developing resilience through emotions, attitudes, \& meaning (gifted edition)-A second wave positive psychology approach. Couns. Psychol. Q. 2019, 32, 307-332. [CrossRef]

31. Barrera Mares, S.A.; Acle Tomasini, G.; Martínez Basurto, L.M. Modelo ecosistémico de riesgo/resiliencia: Validez social de un programa para niños con problemas de lenguaje. In Psicología y Educación: Presente y Futuro, 1st ed.; Castejón Costa, J.L., Ed.; ACIPE: Alicante, Spain, 2016; pp. 2198-2207.

32. Cantero-García, M.; Garrido-Hernansaiz, H.; Alonso-Tapia, J. Diseño del programa de apoyo psicoeducativo para promover la resiliencia parental “Supérate. ¡No tires la toalla!”. Electron. J. Res. Educ. Psychol. 2020, 18, 583-612. [CrossRef]

33. Dray, J.; Bowman, J.; Campbell, E.; Freund, M.; Hodder, R.; Wolfenden, L.; Richards, J.; Leane, C.; Green, S.; Lecathelinais, C.; et al. Effectiveness of a pragmatic school-based universal intervention targeting student resilience protective factors in reducing mental health problems in adolescents. J. Adolesc. 2017, 57, 74-89. [CrossRef]

34. Jenkins, E.K.; Bungay, V.; Patterson, A.; Saewyc, E.M.; Johnson, J.L. Assessing the impacts and outcomes of youth driven mental health promotion: A mixed-methods assessment of the Social Networking Action for Resilience study. J. Adolesc. 2018, 67, 1-11. [CrossRef]

35. Li, X.; Harrison, S.E.; Fairchild, A.J.; Chi, P.; Zhao, J.; Zhao, G. A randomized controlled trial of a resilience-based intervention on psychosocial well-being of children affected by HIV/AIDS: Effects at 6- and 12-month follow-up. Soc. Sci. Med. 2017, 190, 256-264. [CrossRef] [PubMed]

36. Morote, R.; Las Hayas, C.; Izco-Basurko, I.; Anyan, F.; Fullaondo, A.; Donisi, V.; Zwiefka, A.; Gudmundsdottir, D.G.; Ledertoug M.M.; Olafsdottir, A.S.; et al. Co-creation and regional adaptation of a resilience-based universal whole-school program in five European regions. Eur. Educ. Res. J. 2020. [CrossRef]

37. Muñoz Garrido, V. La resiliencia: Una intervención educativa en pedagogía hospitalaria. Rev. Educ. Inclusiva 2016, 9, 79-89.

38. Pérez Quinteros, L.E. La resiliencia en estudiantes con Déficit Atencional por Hiperactividad. Rev. Int. Apoyo Incl. Logop. Soc. Multicult. 2018, 4, 111-123. [CrossRef]

39. Rascón Gómez, M.T.; Cabello Fernández-Delgado, F. Narrativas audiovisuales sobre resiliencia y educación desde un enfoque edu-comunicativo. Innov. Educ. 2019, 19, 77-92.

40. Rosen, J.G.; Phiri, L.; Chibuye, M.; Namukonda, E.S.; Mbizvo, M.T.; Kayeyi, N. Integrated psychosocial, economic strengthening, and clinical service-delivery to improve health and resilience of adolescents living with HIV and their caregivers: Findings from a prospective cohort study in Zambia. PLOS ONE 2021, 16, e0243822. [CrossRef]

41. Saltzman, W.R. The FOCUS Family Resilience Program: An Innovative Family Intervention for Trauma and Loss. Fam. Process 2016, 55, 647-659. [CrossRef]

42. Sánchez-Hernández, Ó.; Méndez Carrillo, F.X.; Garber, J. Promoting resilience in children with depressive symptoms | Promoción de la resiliencia en niños con sintomatología depresiva. An. Psicol. 2016, 32, 741-748. [CrossRef]

43. Saul, J.; Simon, W. Building Resilience in Families, Communities, and Organizations: A Training Program in Global Mental Health and Psychosocial Support. Fam. Process 2016, 55, 689-700. [CrossRef]

44. Joint Committee on Standards for Educational Evaluation. Standards for Evaluations of Educational Programs, Projects, and Materials; McGraw-Hill: New York, NY, USA, 1994.

45. Falicov, C.J. Training to Think Culturally: A Multidimensional Comparative Framework. Fam. Process 1995, 34, $373-388$. [CrossRef]

46. Grau Rubio, C. Fomentar la resiliencia en familias con enfermedades crónicas pedriátricas. Rev. Española Discapac. 2013, 1, 195-212. [CrossRef]

47. Rosselló, M.R.; Verger, S.; Negre, F.; Lourido, B.P. Interdisciplinary care for children with rare diseases. Nurs. Care Open Access J. 2018, 5, 11-12. [CrossRef]

48. Boingboing. Resilience Framework (Children \& Young People), October 2012-Adapted from Hart \& Blincow 2007 [Internet]. 2012. Available online: https://www.boingboing.org.uk/wp-content/uploads/2017/02/resilience-framework-children-andyoung-people-2012-spanish-final.pdf (accessed on 7 March 2019). 